\title{
Bifurcations and chaos in single-roll natural convection with low Prandtl number
}

\author{
I. Mercader, O. Batiste, and L. Ramírez-Piscina \\ Departament de Física Aplicada, Universitat Politècnica de Catalunya, Jordi Girona 1-3, Campus Nord, \\ Módul B4, E-08034 Barcelona, Spain \\ X. Ruiz \\ Física Aplicada, Universitat Rovira i Virgili, Marcel i Domingo s/n, Campus Sant Pere Sescelades, \\ E-43005 Tarragona, Spain \\ S. Rüdiger \\ Hahn-Meitner Institute, Glienicker Strasse 100, D-14109 Berlin, Germany \\ J. Casademunt \\ Departament d'Estructura i Constituents de la Matèria, Universitat de Barcelona, Diagonal 647, \\ E-08028 Barcelona, Spain
}

(Received 30 March 2005; accepted 14 September 2005; published online 25 October 2005)

\begin{abstract}
Convective flows of a small Prandtl number fluid contained in a two-dimensional cavity subject to a lateral thermal gradient are numerically studied by using different techniques. The aspect ratio (length to height) is kept at around 2. This value is found optimal to make the flow most unstable while keeping the basic single-roll structure. Two cases of thermal boundary conditions on the horizontal plates are considered: perfectly conducting and adiabatic. For increasing Rayleigh numbers we find a transition from steady flow to periodic oscillations through a supercritical Hopf bifurcation that maintains the centrosymmetry of the basic circulation. For a Rayleigh number of about ten times that of the Hopf bifurcation the system initiates a complex scenario of bifurcations. In the conductive case these include a quasiperiodic route to chaos. In the adiabatic one the dynamics is dominated by the interaction of two Neimark-Sacker bifurcations of the basic periodic solutions, leading to the stable coexistence of three incommensurate frequencies, and finally to chaos. In all cases, the complex time-dependent behavior does not break the basic, single-roll structure. () 2005 American Institute of Physics. [DOI: 10.1063/1.2107907]
\end{abstract}

\section{INTRODUCTION}

The flux produced in confined fluids by differential lateral heating has received growing interest as a prototype problem to deepen our fundamental understanding of instabilities leading to unsteady flows. Despite its apparent simplicity, the problem has already revealed a rich variety of complex behaviors and a rather strong sensitivity to small variations of parameters, such as the aspect ratio (length over height) or the Prandtl number, and to the boundary conditions (both thermal and mechanical). ${ }^{1-11}$ From the applied point of view, the phenomenon of natural convection is ubiquitous. As opposed to the Rayleigh-Bénard convection, the quiescent state is not a solution; consequently, fluid motion is present without the need to overcome a threshold value of any parameter. Matter and energy transport in real processes are very often driven by this kind of convection, which makes it most interesting in a large diversity of applied fields.

On the other hand, it is well known that macroscopic properties and performance of solidified materials depend to a large extent on its microstructure, which in turn is determined by the conditions in which the solid was grown. ${ }^{12}$ For example, instabilities of the melt flow during solidification have been identified as the origin of compositional striations in semiconductor crystals. ${ }^{13}$ These facts have boosted in the past years the interest of the crystal growth community in the complex aspects of the behavior of the fluid flow. Here we focus on configurations relevant to the solidification of diluted alloys by the Bridgman method. In particular, we will consider horizontal rectangular cavities of the moderate aspect ratio and small values of the Prandtl number, proper of metallic and semiconductor melts.

The behavior of the flow inside differentially heated cavities with an aspect ratio equal to 4 has been widely investigated in the literature, partially because it corresponds to the well-known GAMM (Gesellschaft für Angewandte Mathematik und Mechanik) benchmark problem for small Prandtl fluids. ${ }^{14}$ In particular, the primary Hopf bifurcation of the base single-roll solution has extensively been studied both in two-dimensional (2D) (Ref. 3) and three-dimensional (3D) (Refs. 4-10) flows. Secondary instabilities have also been studied both experimentally ${ }^{9}$ and numerically. ${ }^{10}$ The problem of varying the aspect ratio in a systematic way has been addressed only rather recently, ${ }^{11}$ showing the coexistence of steady solutions with different number of rolls. However our interest here is the study of instabilities leading to complex temporal behaviors of the basic single-roll convective flow, rather than instabilities that break the basic roll into several roll configurations (favored by large aspect ratios) or 3D instabilities. Here we will thus present an exhaustive nu- 
merical study of the 2D version of the problem, at a relatively small aspect ratio. The study proposed is intended to gain insight into the type of instabilities and complex timedependent phenomena to be expected in the problem, but the extent to which the results could be extrapolated to three dimensions is uncertain. While the dynamical scenarios encountered in two dimensions will always be present in the full 3D problem, new instabilities may appear in the transversal direction that could eventually dominate. ${ }^{9,10}$ The elucidation of the interplay between 2D and 3D instabilities as a function of other parameters is clearly beyond the scope of this work.

A first series of simulations will study the first Hopf bifurcation by varying both the Prandtl number (in the range of small values) and aspect ratio (in the range permitting single roll solutions). A conclusion drawn from these results is that the aspect ratio 2 is optimal, in a sense that will be made precise, to favor the instability of the flow while keeping the single-roll structure. This value of the aspect ratio, and the Prandtl number corresponding to liquid germanium (as representative of fluids with interest in crystal growth), will be used afterwards to span a large range of variation of the Rayleigh number to elucidate the complex bifurcation scenario of successive instabilities and routes to chaos. A second focus of interest is on the role of boundary conditions at the top and bottom plates. We have carried out a detailed comparative study of the dynamics for two limiting cases of boundary conditions, namely, adiabatic and perfectly conductive walls.

The layout of this paper is as follows. In Sec. II we present the equations for the system and describe the symmetries of the problem and the different numerical schemes employed. Section III is devoted to analyze the basic state and its primary bifurcation. In Sec. IV, the main body of the paper, we describe in detail time-dependent solutions, both periodic and aperiodic, for the two thermal boundary conditions considered in the work. Finally, in Sec. V we present a brief summary of the main results and some concluding remarks.

\section{BASIC EQUATIONS, SYMMETRIES, AND NUMERICAL METHODS}

We have considered an incompressible fluid in a 2D rectangular cavity of aspect ratio $\Gamma=d / h$, with $d, h$ being its length and height, respectively. In the presence of a vertical constant gravity, a temperature difference $\Delta T$ is horizontally maintained over the length of the cell; with the temperature on the right wall higher than that on the left. If we nondimensionalize the equations using $h$ as unit length and the vertical thermal diffusion time $t_{T}=h^{2} / \kappa$ as unit time (being $\kappa$ the thermal diffusivity), the dimensionless equations in the Boussinesq approximation read

$$
\begin{aligned}
& \nabla \cdot \mathbf{u}=0, \\
& \partial_{t} \mathbf{u}+(\mathbf{u} \cdot \nabla) \mathbf{u}=-\nabla P+\sigma \nabla^{2} \mathbf{u}+\sigma R a[-0.5+x / \Gamma+\theta] \mathbf{z},
\end{aligned}
$$

$$
\partial_{t} \theta+(\mathbf{u} \cdot \nabla) \theta=-u / \Gamma+\nabla^{2} \theta .
$$

Here $\mathbf{u} \equiv(u, w)$ is the dimensionless velocity field in $(x, z)$ coordinates, $P$ is the pressure over the density, and $\theta$ denotes the departure of the temperature from the constant-gradient profile in the horizontal direction in units of the imposed temperature difference $\Delta T$. The origin of coordinates is taken at the left, low corner of the rectangular domain, which then spans the intervals $(0, \Gamma)$ and $(0,1)$ in the $x$ and $z$ axes, respectively.

The relevant dimensionless parameters of the flow are the Prandtl number, $\sigma$, and the Rayleigh number, Ra, defined as

$$
\sigma=\frac{\nu}{\kappa}, \quad \mathrm{Ra}=\frac{\alpha g h^{3}}{\nu \kappa} \Delta T
$$

where $\nu$ denotes the kinematic viscosity, $g$ the gravity acceleration, and $\alpha$ the thermal expansion coefficient.

No-slip boundary conditions are used in all the walls of the cavity, and the right and left ones are maintained at constant temperatures, i.e.,

$$
\begin{aligned}
& \mathbf{u}=0, \quad \text { at } \partial \Omega, \\
& \theta=0, \quad \text { at } x=0, \quad \Gamma .
\end{aligned}
$$

Two distinct cases are considered for the boundary conditions for the temperature at the horizontal lids. For the first one, the perfectly conducting case, a linear profile between the two prescribed temperatures is imposed. The second one, the adiabatic case, corresponds to imposing no heat flux boundary conditions. For the dimensionless temperature $\theta$ the conditions are

$$
\begin{aligned}
& \theta=0, \quad \text { at } z=0,1 \quad \text { (perfectly conducting case), } \\
& \partial_{z} \theta=0, \quad \text { at } z=0,1 \quad \text { (adiabatic case). }
\end{aligned}
$$

As a measure of the relative heat transported by convection, we will use the Nusselt number $\mathrm{Nu}$ evaluated at the cooler wall of the cavity. This corresponds to computing the ratio of the total heat flux through the left wall to the total conductive flux that would correspond to the case of a linear temperature profile; that is, for the same setup but without gravity. Thus, the Nusselt number has the following expression:

$$
\mathrm{Nu}=1+\Gamma\left\langle\partial_{x} \theta\right\rangle_{x=0},
$$

where the symbol \langle\rangle$_{x=0}$ denotes the spatial average over the left sidewall. We will also evaluate, as an estimate of the strength of the convection, the dimensionless quantity $E_{K}$, defined by

$$
E_{K}=\frac{1}{\Gamma} \int_{x=0}^{x=\Gamma} \int_{z=0}^{z=1} \mathbf{u} \cdot \mathbf{u} d x d z,
$$

which is directly related to the mean kinetic energy of the system.

Equations (1), together with boundary conditions (3), are invariant under the transformation operator 


$$
R:(x, z) \rightarrow(\Gamma-x, 1-z), \quad(u, w, \theta) \rightarrow(-u,-w,-\theta),
$$

i.e., a rotation of an angle $\pi$ around the center point $(\Gamma / 2,1 / 2)$. That is, if $\Psi=(u, w, \theta)$ is a solution of the problem, $R \Psi$ will also be a solution. Two solutions related by the $R$ transformation are called $R$ conjugated. Notice that the Nusselt number $\mathrm{Nu}$ as defined above will be, in general, different for two conjugated solutions, whereas the kinetic energy $E_{K}$ will be the same.

Since $R^{2}=I$, the transformation $R$ is a generalized reflection, and the resulting symmetry group is $\mathbf{Z}_{2}=\{I, R\}$. Invariant solutions under $R$, i.e., $R \Psi_{f}(t)=\Psi_{f}(t)$ for all $t$, are named $R$ equivariant or fixed solutions. ${ }^{15}$ If $\Psi(t)$ is a periodic fixed solution, the associated periodic orbit in the phase space is called an $F$ cycle. Periodic solutions, with period $T$, that verify the spatiotemporal symmetry $R \Psi_{s}(t)=\Psi_{s}(t+T / 2)$, are called symmetric and the associated periodic orbit is called an $S$ cycle. The cycles corresponding to a pair of $R$-conjugated symmetric solutions are identical. Symmetric solutions are typically found in problems with periodic forcing. ${ }^{16}$ Codimension 1 bifurcations of equilibria and fixed and symmetric periodic solutions in $\mathbf{Z}_{2}$ equivariant systems are discussed in detail in Ref. 15. It is worth mentioning that the $R$ symmetry inhibits the period-doubling bifurcation of an $S$ cycle (Floquet multiplier $\mu=-1$ ), whereas this bifurcation is allowed for an $F$ cycle.

For the bulk of the study of this system we have solved the evolution equations (1) and boundary conditions (3) using a second-order time-splitting algorithm, proposed in Ref. 17, with a pseudospectral Chebyshev method for the spatial discretization. This algorithm has been used previously by some of the authors to study binary mixtures. ${ }^{18-20}$ The method employs a pressure boundary condition, which in conjunction with stiffly stable schemes prevents propagation and accumulation of time differencing errors. ${ }^{21}$ On Chebyshev collocation points, the Helmholtz and Poisson equations resulting from the time splitting are solved efficiently by using a complete diagonalization of operators in both directions. ${ }^{22}$ For values of the Rayleigh number that are not too high, a second-order structured finite volume code based on the SIMPLE algorithm to couple the transport equations ${ }^{23}$ has also been used.

To calculate steady solutions we have adapted a pseudospectral first-order time-stepping formulation to carry out Newton's method, as described in Refs. 4, 24, and 25. In the preconditioned version of Newton's iteration, the corresponding linear system is solved by an iterative technique using a GMRES package. ${ }^{26}$ The right-hand side of the preconditioned linear system corresponds to perform one time step of the full nonlinear equations, and the left-hand side corresponds to one time step of the linearized equations, so the Jacobian matrix is never constructed or stored.

Regarding the linear stability analysis of the steady states, Arnoldi's method has been applied to calculate the dominant eigenvalues of the exponential of the Jacobian, which can be trivially related with the leading eigenvalues (i.e., with the largest real part) of the Jacobian. To this end, the algorithm for time stepping of the linearized equations has been used, since in fact it approximates the action of the exponential transformation of the Jacobian on the solution at the previous time step. The eigensolving itself has been implemented by using the ARPACK package.

For some purposes we have also used a spectral Galerkin method with the basis functions being the so-called Chandrasekhar functions. The resulting system of ordinary differential equations has then been studied with the standard software package AUTO for path-following continuation techniques.

For the range of parameters considered in this paper, around $\Gamma=2$ and Prandtl number $\sigma=0.00715$, the resolution needed for good accuracy depended, as usual, on the value of the Rayleigh number. For both of the used boundary conditions, to calculate steady solutions $\left(\mathrm{Ra}<2.0 \times 10^{4}\right)$ a resolution of $N_{x}=30 \times N_{z}=30$ sufficed to give more than three significant figures in the value of the Nusselt number. To compute the time-dependent solutions by the pseudospectral method at Rayleigh numbers higher than $2 \times 10^{4}$ we have used $N_{x}=90 \times N_{z}=60$ and a time step $\Delta t=10^{-4}$. Computations with a larger resolution $(120,90)$ and smaller time step $\Delta t=5 \times 10^{-5}$ have been carried out regularly to ensure that no significant changes were observed in the solutions. As an illustration of the precision reached by using these resolutions, the basic frequency of the frequency locking (1:12) obtained at $\mathrm{Ra}=22900$ in the conductive case gave the same value of 3.6391 by using these two resolutions, while with a resolution of only $N_{x}=72 \times N_{z}=48$ and $\Delta t=5 \times 10^{-5}$ it gave a value of 3.6420 .

The finite volume method was employed in cases with Rayleigh numbers lower than $2 \times 10^{4}$, for which a 9600 $(121 \times 81)$ control volume distribution correctly reproduced pseudospectral results. However, for larger Rayleigh numbers the needed number of volumes increases in such a way that computation becomes extremely heavy and the method is not suitable. With respect to the mentioned spectral Galerkin calculations, $20 \times 20$ basis functions were necessary for results to reasonably converge, reaching agreements better than $\sim 3 \%$ for $\operatorname{Ra}_{c}$ and $\omega_{c}$, until Rayleigh numbers of the order of 5000 for $\Gamma=2$. Beyond this, the increased number of variables resulting from the necessary increase in resolution made impractical the use of the AUTO package.

Note that, in principle, the time integration used in most of this work allows only for the finding of stable solutions. The use of a Newton method has permitted to obtain also some unstable solutions, but only out of the steady branch. A more complete bifurcation analysis would require the calculation of unstable orbits, but this is far beyond the scope of the present work. In any case, this could be achieved, for instance, by means of the methods employed in Refs. 27-29. Also the AUTO package could in principle have been used for this purpose. However, interesting temporal behavior appears here in regimes where such computation is extremely costly, since large numbers of modes must be taken into consideration to properly resolve the system and achieve sufficient accuracy, as commented above. This has prevented us from using such methods for the present problem. 
$T$

a)

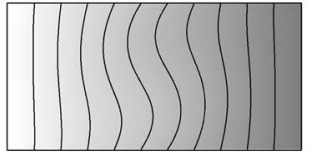

$\psi$

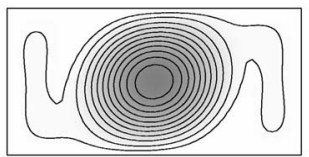

$\mathrm{T}$

b)

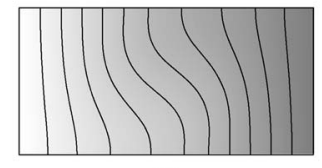

$\psi$

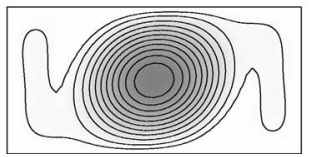

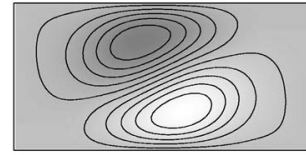

$\omega$

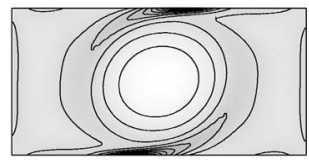

$\theta$

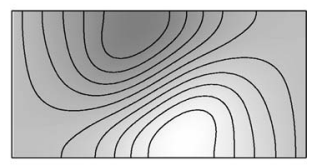

$\omega$

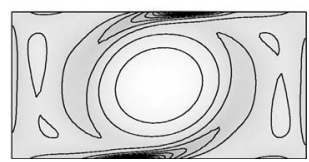

FIG. 1. Contour plots of temperature $T$, deviation of the temperature $\theta$, stream function $\Psi$, and vorticity $\omega$, for the steady solutions corresponding to (a) the perfectly conducting case $(\mathrm{Ra}=1950)$ and $(\mathrm{b})$ the adiabatic case $(\mathrm{Ra}=2300)$.

\section{BASIC STATE AND PRIMARY BIFURCATION}

In the remainder of the paper we present results mainly corresponding to a fluid with Prandtl number $\sigma=0.00715$ contained in a rectangular cavity of aspect ratio $\Gamma=2$. For this selected case and the two chosen boundary conditions we will explore a large range of Rayleigh numbers. As we will show in the next section, even with such a small Prandtl number, the dynamics obtained depends on the thermal conditions used at the walls. In the present section we analyze the basic state and the primary bifurcation. We will also follow the variations of this instability when changing the parameters $\sigma$ and $\Gamma$.

For moderate Rayleigh numbers $\left(\mathrm{Ra} \approx 10^{3}\right)$, the only stable solution is steady and $R$ equivariant, and consists of a single roll. ${ }^{11}$ As the Rayleigh number increases, this roll concentrates in the center, tilts in the direction of one of the diagonals, and two stagnation points are generated giving rise to two weakly corotating circulations in the sides of the cavity, as can be seen in Fig. 1. In this figure we show the isotherms, the streamlines (contours of the stream function $\Psi$, defined by $u=\partial_{z} \Psi$ and $\left.w=-\partial_{x} \Psi\right)$, the contours of the deviation of the temperature with respect to the linear profile, $\theta$, and the vorticity $\left(\omega_{y}\right)$, for steady solutions corresponding to both the perfectly conducting case $(\mathrm{Ra}=1950)$ and the adiabatic case $(\mathrm{Ra}=2300)$. For these two values of the Rayleigh number, solutions are close to becoming unstable. In these figures, darker zones indicate higher values of the represented fields (note that the rolls are counterclockwise). For both of the considered boundary conditions, the steady solution loses stability at a supercritical Hopf bifurcation that maintains the $R$ symmetry of the basic solution, giving rise to a fixed periodic solution ( $F$ cycle). The values of the critical Rayleigh number $\mathrm{Ra}_{c}$ and Hopf angular frequency $\omega_{c}$ are
$\theta(\mathrm{Re})$

a)

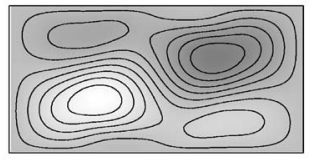

$\psi(\operatorname{Re})$

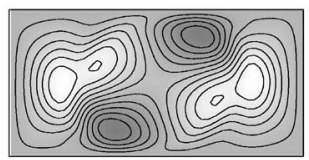

$\theta(\mathrm{Re})$

b)

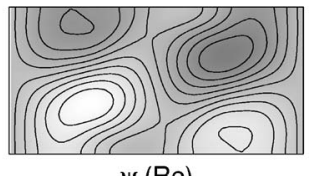

$\psi(\mathrm{Re})$

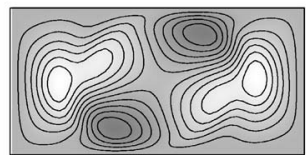

$\theta(\mathrm{Im})$

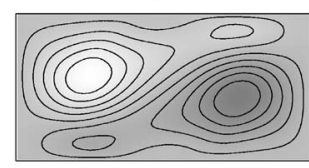

$\psi(\operatorname{lm})$

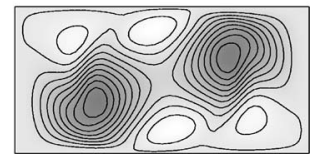

$\theta(\mathrm{Im})$

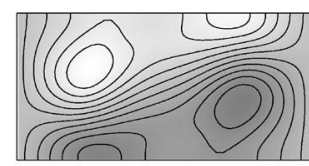

$\psi(\operatorname{lm})$

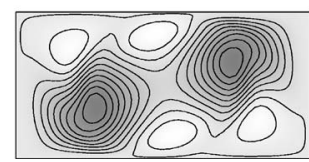

FIG. 2. Real and imaginary parts of the eigenfunctions (contours of $\theta$, stream function) of the most unstable mode at the Hopf bifurcation for (a) the perfectly conducting case $\left(\mathrm{Ra}_{c}=1.991 \times 10^{3}\right)$ and (b) the adiabatic case $\left(\mathrm{Ra}_{c}=2.381 \times 10^{3}\right)$.

$\mathrm{Ra}_{c}=1.991 \times 10^{3}, \omega_{c}=6.03$ for the perfectly conducting case, and $\mathrm{Ra}_{c}=2.381 \times 10^{3}, \omega_{c}=6.04$ for the adiabatic case. In Fig. 2 we have plotted the real and imaginary parts of the eigenfunctions (deviation of temperature, stream function) of the unstable mode at the bifurcation point for both cases. Notice that, except in the behavior of the deviation of temperature near the horizontal plates, eigenfunctions are nearly identical for both cases. We can conclude that for this small Prandtl number, this first instability is roughly independent of thermal boundary conditions, and is rather of hydrodynamical nature, probably associated with the strong shear present near the horizontal walls. Consistently, the Hopf frequency is almost the same in both cases. Furthermore, since shear is stronger in the perfectly conducting case at a given value of Rayleigh number, the instability occurs in this case for a smaller value of this number. This instability hardly affects the core of the roll and produces a periodic motion stretching first the roll in the diagonal direction and getting it back to a nearly circular shape. When the roll stretches, a larger warm (cold) region settles at the left (right) part of the diagonal. During this cycle small vortices appear and disappear at the corners of the cavity.

To determine the sensitivity of the dynamics to the two parameters $\sigma$ and $\Gamma$, which remain unchanged in the rest of the study, we have calculated the primary solutions and their stability for these parameters varying in a neighborhood of the reference values used in the other numerical simulations.

Figure 3(a) shows the dependence of the threshold for the first Hopf bifurcation on the Prandtl number. The numerical results show that the Hopf point is moved to a larger Rayleigh number almost linearly with an increasing Prandtl number, which is consistent with the hydrodynamic origin of this first instability. In fact, the Grashof number (defined as 

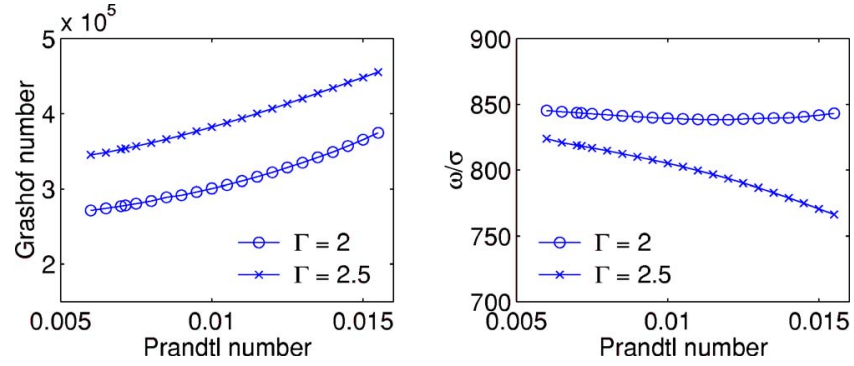

FIG. 3. Critical Grashof number $\mathrm{Gr}=\mathrm{Ra} / \sigma$ (left) and scaled frequency $\omega / \sigma$ for the first Hopf bifurcation as a function of the Prandtl number $\sigma$ for two different aspect ratios $(\Gamma=2$ and $\Gamma=2.5)$.

$\mathrm{Gr}=\mathrm{Ra} / \sigma$ ) measures the ratio of the buoyancy force to the viscous force, and is expected to be the relevant dimensionless parameter in the limit of small Prandtl number. Since in this regime viscous dissipation dominates over thermal dissipation, the critical Grashof number should be independent of the Prandtl number. This should be strictly valid in the limit of vanishing $\sigma$, and it is roughly satisfied in our case, as can be seen in Fig. 3(a). The two curves of this figure represent the critical values of the Grashof number corresponding to the aspect ratios $\Gamma=2$ and $\Gamma=2.5$. This indicates that, while buoyancy is the driving force that sets the flow, the balance between inertia and momentum diffusion is the actual source of instability. Furthermore, Fig. 3(b) shows the corresponding Hopf frequency, scaled with $\sigma$, for $\Gamma=2$ and $\Gamma=2.5$. There is a roughly linear relation between the Prandtl number and the Hopf frequency within this range. This scaling is clearly shown in Fig. 3(b), where the angular frequency in viscous units $\omega / \sigma$ appears as nearly constant (more distinctly for the case of $\Gamma=2$ ). This means that the critical frequency scales with the momentum diffusion time $h^{2} / \nu$, indicating again that oscillations are governed by the balance between inertia and momentum diffusion, with negligible thermal effects.

In order to test the sensitivity to aspect ratio, we have also determined the critical Rayleigh numbers and the corresponding Hopf frequencies for a set of different aspect ratios keeping $\sigma$ fixed to 0.006 , as shown in Fig. 4. The most salient feature of this figure is the existence of a pronounced minimum of the critical $\mathrm{Ra}$ for an aspect ratio close to $\Gamma$ $=2$. Around this value, the single-roll is most unstable to time-dependent behavior. The corresponding Hopf frequency is also shown in Fig. 4, with a more shallow minimum at higher values of aspect ratio. While the Rayleigh threshold
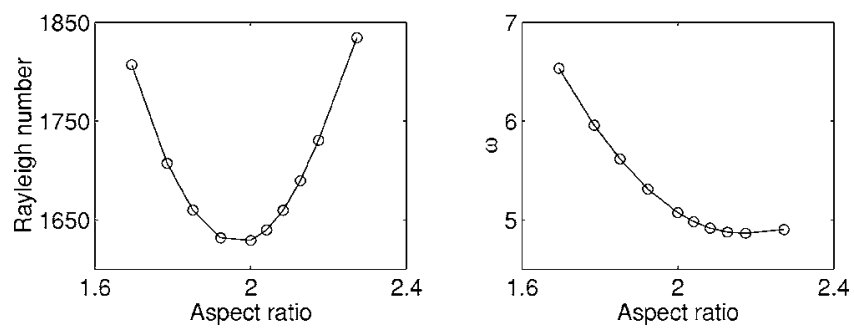

FIG. 4. Critical Rayleigh number and frequency $\omega$ for the first Hopf bifurcation as a function of the aspect ratio for Prandtl number $\sigma=0.006$.
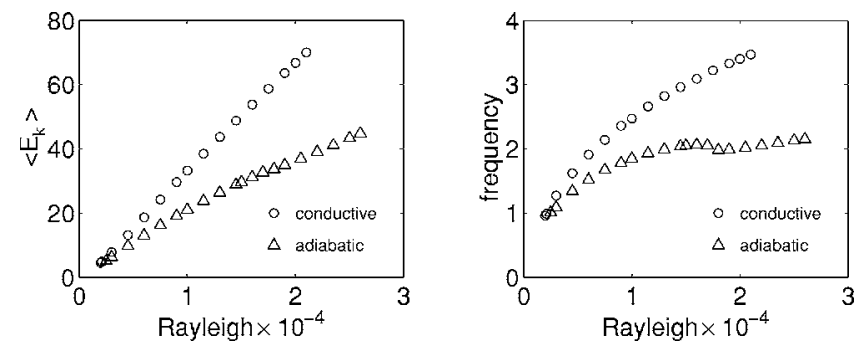

FIG. 5. Mean kinetic energy and frequency as a function of the Rayleigh number, corresponding to the $R$-equivariant stable periodic solutions that bifurcate from the basic steady state. Circles are used for the solutions of the perfectly conducting case and triangles for those of the adiabatic case.

decreases fast with an aspect ratio of $\Gamma<2$, it becomes rather insensitive to $\Gamma$ for moderately larger values.

\section{TIME-DEPENDENT SOLUTIONS}

In the remainder of the paper we will analyze the dynamics of the system when Rayleigh number is increased beyond the Hopf bifurcation. Since solutions have been obtained from a time-dependent code, we have only been able to determine stable solutions. To characterize the temporal dependence of the solutions we have recorded a few local variables, namely, the values of the deviation of temperature $\theta$ and of the two components of velocity $u$ and $w$ at the point $P_{1}=(3 \Gamma / 4,3 / 4)$. As an indicator of the symmetric properties of the solution we have used the value of the local quantity sym defined as the sum of the vertical velocity $w$ in $P_{1}$ and in its centrosymmetric point $P_{2}=(\Gamma / 4,1 / 4)$, i.e., $\operatorname{sym}=w\left(P_{1}\right)$ $+w\left(P_{2}\right)$. For an $R$-equivariant solution, sym is always zero, whereas for a symmetric periodic solution ( $S$ cycle) only its mean value is guaranteed to be so. This latter type of periodic solution can appear in our system through a symmetrybreaking Hopf bifurcation of a steady fixed solution, or through a flip (period-doubling) bifurcation with symmetry breaking of an $F$ cycle (i.e., a periodic fixed solution). Neimark-Sacker bifurcations (Hopf bifurcations of periodic solutions) both for $F$ or $S$ cycles always result in $R$-equivariant two-tori. ${ }^{15}$ That is, for a nonfixed quasiperiodic solution, the orbit associated with the conjugated solution resides on the same torus, and is denoted by $\mathbb{T}^{2}(S)$. The time average (for a long-time interval) of the variable sym for such a solution is also zero.

\section{A. Periodic solutions}

Beyond the primary supercritical Hopf bifurcation, stable $F$ cycles can be found until a Neimark-Sacker bifurcation occurs for both boundary conditions. The values of the Rayleigh number at which the bifurcation occurs are $\mathrm{Ra}_{\mathrm{NS}}$ $\approx 2.11 \times 10^{4}$ for the perfectly conducting case and $\mathrm{Ra}_{\mathrm{NS}}$ $\approx 2.62 \times 10^{4}$ for the adiabatic case. In Fig. 5 we have displayed the kinetic energy $E_{K}$ [Eq. (5)] averaged in a period, $\left\langle E_{K}\right\rangle$, and the frequency $f$ of these cycles as a function of the Rayleigh number. Circles are used for the solutions of the perfectly conducting case and triangles for those of the adiabatic case. This figure shows that, for these values of the Rayleigh number, the different boundary conditions on the 


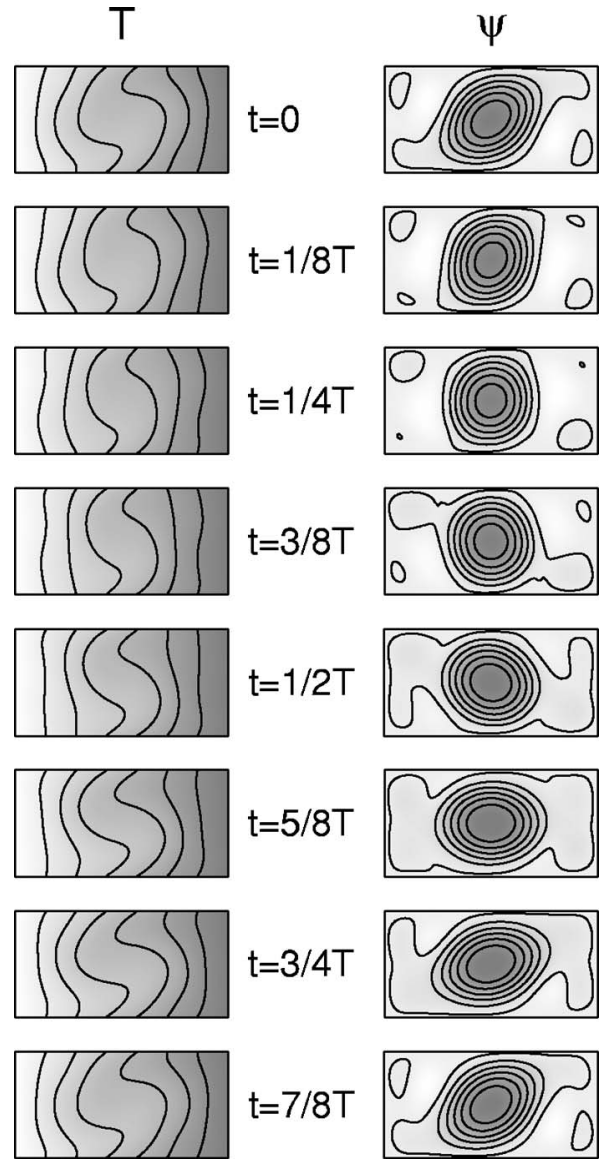

FIG. 6. Time sequence of the temperature field and streamlines of the periodic solution at $\mathrm{Ra}=21000$, for the perfectly conducting case. The period of this solution is $T=0.29$ in units of the vertical thermal diffusion time.

horizontal plates start to have a distinct influence on the dynamics. The value of $\left\langle E_{K}\right\rangle$, as a measure of convection intensity, is higher for the perfectly conducting case, and grows almost linearly with the Rayleigh number. For the adiabatic case, the slope of this curve changes near $\mathrm{Ra}=15000$, while frequencies saturate, and even slightly decrease. In this range of Rayleigh numbers, a close inspection of the temporal evolution during transients (e.g., when the temporal integration is started from a solution obtained with a smaller Ra) reveals the presence of a double-period mode with a rather slow relaxation. However, this period-doubling tendency is not reflected in the final orbit, and the resulting frequencies vary continuously with the Rayleigh number as is shown in Fig. 5. The proximity of a branch of solutions with double period could be the origin of this behavior.

Time sequences spanning one period, showing snapshots of isotherms and streamlines at eight equidistant times, are depicted for two $F$ cycles in Figs. 6 and 7. Figure 6 corresponds to a solution for the conducting case at $\mathrm{Ra}=21000$, with a period $T=0.29$. The behavior of this solution is essentially the result of the Hopf instability described above. Figure 7 shows a periodic solution for the adiabatic case at $\mathrm{Ra}$ $=25000$, with a period $T=0.47$. We can see in this figure that adiabatic boundary conditions break the rigidity of the isotherms allowing for an expansion of the central structure with the resulting reduction of secondary vortices in the cor-

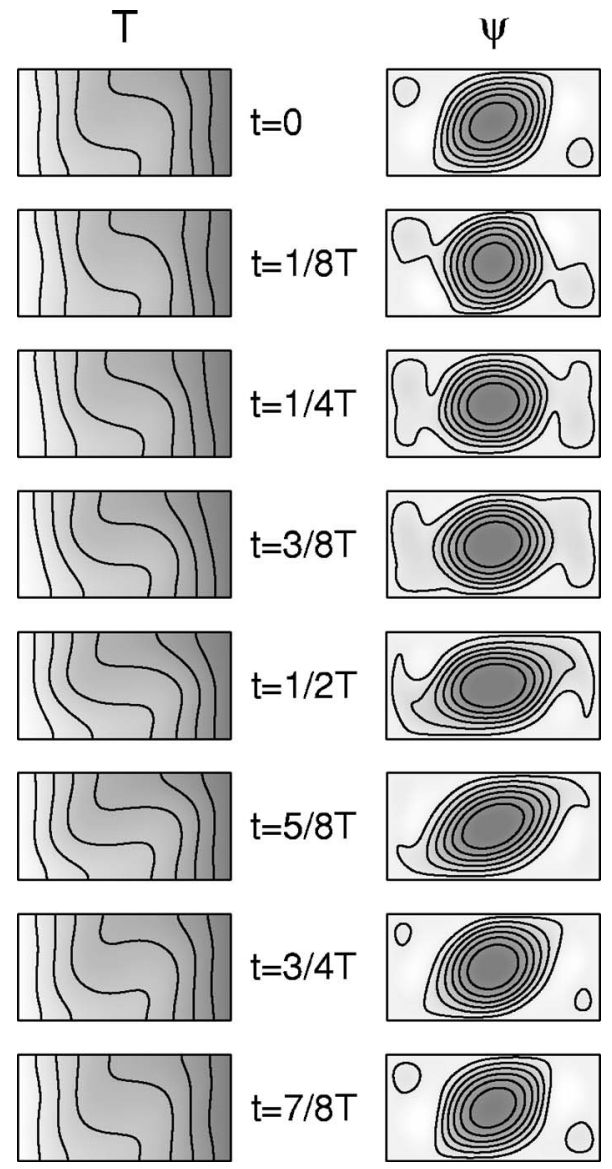

FIG. 7. Time sequence of the temperature field and streamlines of the periodic solution at $\mathrm{Ra}=25000$, for the adiabatic case. The period of this solution is $T=0.47$ in units of the vertical thermal diffusion time.

ners. The temperature field appears more clearly (stably) stratified in the central region of the cavity, i.e., isothermal lines are roughly horizontal with temperature increasing in the vertical direction. A stratification of this type could induce the generation of internal gravity waves as those responsible for the oscillatory instability in air $(\sigma=0.71)$ (Refs. 1 and 2). This will be further discussed below.

\section{B. Secondary bifurcations}

As stated above, the $F$ cycles lose stability via NeimarkSacker bifurcations. As thermal effects become increasingly important, these secondary bifurcations present distinct features for both the conductive and the adiabatic cases, but they do seem to share common mechanisms.

For the conductive case, the Neimark-Sacker bifurcation occurs at $\mathrm{Ra}_{\mathrm{NS}} \approx 2.11 \times 10^{4}$. The bifurcation is subcritical in this case, and the eigenvectors $\xi_{i}$, corresponding to the complex multipliers $\mu_{i}=e^{ \pm i \theta_{0}}$ of the Jacobian matrix of the Poincaré map associated with the cycle, verify $R \xi_{i}=-\xi_{i}$. Thus, the quasiperiodic solution that bifurcates from the $F$ cycle is no longer $R$ equivariant. This instability, with the unstable mode breaking the symmetry of the base solution, could be difficult to detect in numerical time integration as discussed in Ref. 4. This is due to the fact that perturbations have to grow out of numerical noise and, being close to the 
instability threshold, it is necessary to integrate the evolution equations during an extremely long time in order for the instability to manifest itself. To overcome this problem, we have monitored the value of the variable sym, which is zero for the basic symmetric periodic solution. When the instability triggers on, we can easily detect its growth however small it is. The frequency $f_{2}$ of this subcritical secondary Hopf bifurcation can be inferred from the transient growing oscillations of the variable sym near the bifurcation, when the initial condition is an $R$-equivariant solution. This secondary Hopf frequency $f_{2}$ is approximately $f_{2} \approx 0.30$.

In the adiabatic case the system undergoes the NeimarkSacker bifurcation at $\mathrm{Ra}_{\mathrm{NS}} \approx 2.62 \times 10^{4}$. Contrary to the conducting case, this bifurcation is now supercritical and the eigenvectors $\xi_{i}$ of the Jacobian matrix responsible for the bifurcation verify $R \xi_{i}=\xi_{i}$. Thus, the quasiperiodic solutions which bifurcate from the $F$-cycle branch are $R$ equivariant. The secondary Hopf frequency is $f_{2} \approx 0.43$.

To gain further insight into the physical nature of these secondary instabilities, when a different frequency arises, we have undertaken stroboscopic flow decompositions of the bifurcated solutions, as shown in Figs. 8 and 9. In these figures the evolution given by the new, smaller frequency, can be visualized while the rapid variations of the base cycle are hidden. In the conductive case, as shown below, there are frequency locking windows. This can be exploited to easily construct such stroboscopic decomposition, by subtracting the average flow and depicting the deviation from it at times chosen with the periodicity of the fast oscillation. In addition, since this secondary instability is subcritical, the amplitude of such deviation is relatively large. The analysis of the adiabatic case is more delicate, not only because the frequencies are not commensurate, but also because the instability is supercritical and the corresponding quasiperiodical behavior occurs in a relatively narrow window of Ra. In order to define a stroboscopic map in this case, while presenting equispaced snapshots during the slow period (to properly calculate the average), we have approximated the ratio between frequencies with an appropriate rational number, and chosen the times accordingly. In this way we have obtained the evolution shown in Fig. 9, which could be considered as approximately stroboscopic but which appears as periodic in the slow frequency.

In the stroboscopic decomposition of the conductive case, Fig. 8, the perturbation has the form of two central rolls which exchange the sense of rotation periodically, implying vertical, oscillatory displacement of the fluid in the central region. The center of the cell is also periodically overheated and underheated with the same frequency. In the case of adiabatic conditions, shown in Fig. 9, the perturbed flows look quite different, reflecting the importance of the thermal boundary conditions for the instability. The occurrence of two-roll structures is now located at the corners, producing a similar overheating and underheating but now at those same corners. The different capability of heat exchange through the horizontal boundaries of the cavity plays a clear role here. While in the conductive case heat can be exchanged in
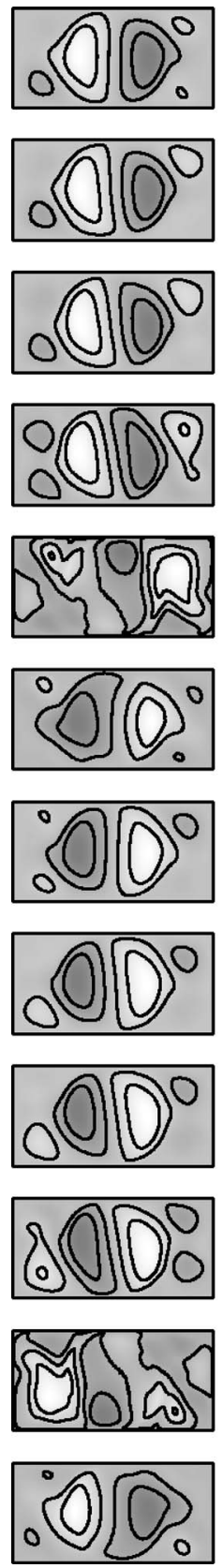
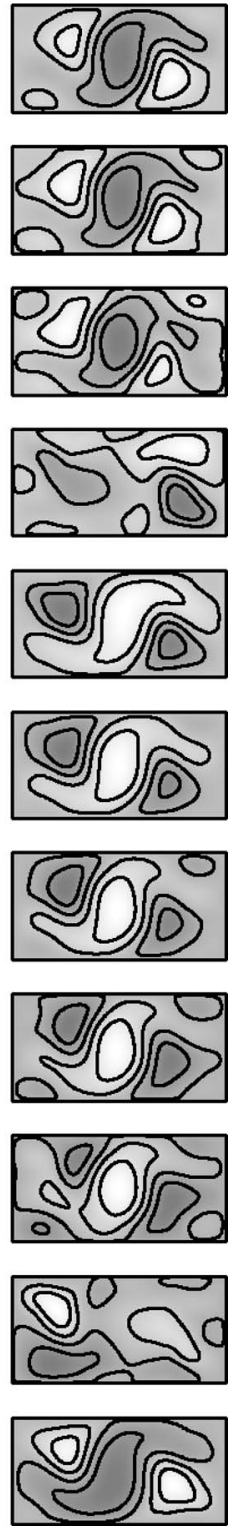
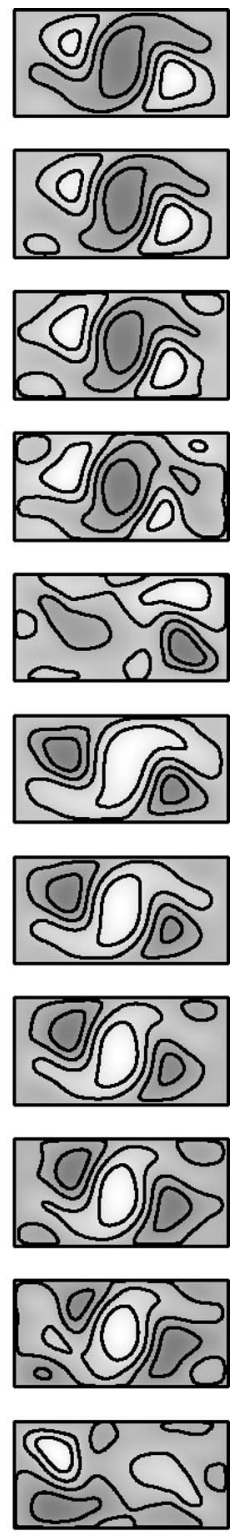

FIG. 8. Perturbation associated with the secondary instability for the conductive case, at $\mathrm{Ra}=22$ 900. Streamlines (left) and temperature fields (right) are represented at equispaced times, multiples of the periodicity of the fast frequency (with a value of 3.6391) in the frequency locking window 1:12 (see the text).

the vertical direction through the horizontal walls, the heat exchanged in the adiabatic case is restricted to the vertical sidewalls.

These results seem to reinforce the conclusion that internal gravity waves may be at the root of these secondary instabilities. The presence of a stable stratification and the actual vertical oscillations of the flow captured by the stroboscopic decomposition do suggest this conclusion. The estimation of the Brunt-Väisälä frequency [computed from the density gradient at the cavity center as $\left(\operatorname{Ra} \sigma \partial_{z} \theta\right)^{1 / 2} / 2 \pi$ in our units] results in values of the order of 1.5, which in principle provides an upper bound for the internal waves frequency. 

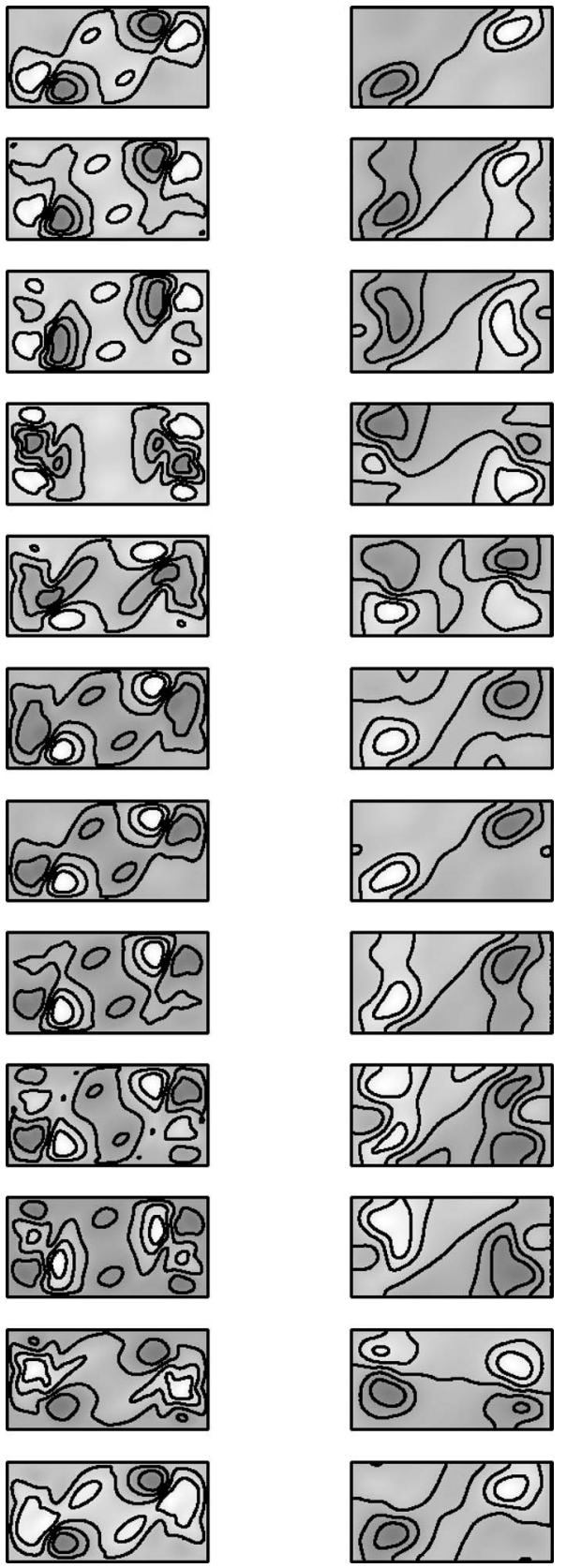

FIG. 9. Perturbation associated with the secondary instability for the adiabatic case, at $\mathrm{Ra}=26$ 700. Streamlines (left) and temperature fields (right) are represented at times chosen by approximating the quasiperiodic solution by an approximate frequency locking 4:25 (see the text). The real (incommensurate) frequencies are $f_{1}=2.1624$ and $f_{2}=0.3435$.

These values are therefore consistent with the internal waves scenario (within the correct order of magnitude), but the finite size of the system and the nonlinear interactions present (dealing with a secondary bifurcation of a nonquiescent base state) make this estimation nonconclusive by itself. Nevertheless, one can find additional evidence in the observation of the dependence of the primary frequency with Rayleigh number, as shown in Fig. 5, in the range of Ra where the secondary frequency emerges. Although the behavior near the first instability is insensitive to boundary conditions, the further increasing of Ra acts differently for both cases, indicating that the stratification could play a significant role in
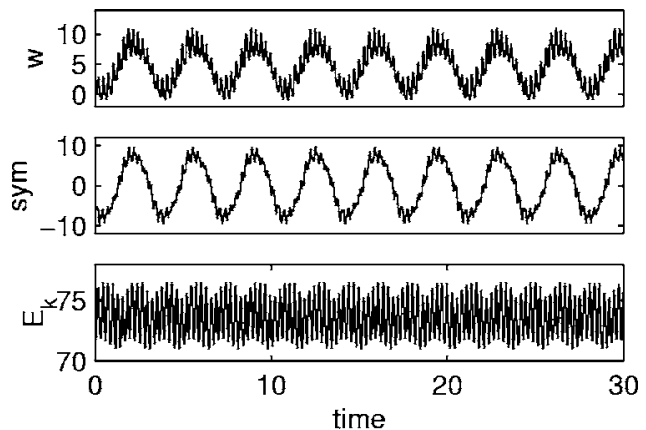

FIG. 10. Time series of the vertical component of the velocity $w$ at the point $P_{1}$, the variable sym, and the kinetic energy $E_{K}$ for the quasiperiodic solution at $\mathrm{Ra}=22000$.

the dynamics of the system in this regime. Indeed, the apparent saturation of the frequency with $\mathrm{Ra}$ in the adiabatic case can be associated with the fact that the stratification of the central region is already built and becomes roughly insensitive to Ra. On the contrary, the conductive boundary conditions force the isotherms to be more contorted in order to produce a stable stratification at the center and simultaneously fulfill the thermal boundary condition at the horizontal walls. As a consequence, the degree of stratification, which is less pronounced in this case (and happens to be locally unstable in some regions), is also more sensitive to $\mathrm{Ra}$. This is consistent with a main role of the stratification in this range of parameters, and hence is also an indication that the origin of the secondary instabilities may indeed be associated with the occurrence of internal gravity waves.

\section{Complex behavior}

\section{Perfectly conducting case}

When the Rayleigh number is increased beyond the Neimark-Sacker bifurcation in the conducting case, we obtain a branch of quasiperiodic states. In Fig. 10 we have plotted the time series corresponding to the vertical component $w$ of the velocity at the point $P_{1}$, the variable sym, and the kinetic energy $E_{K}$, for the quasiperiodic solution at Ra $=22000$. Notice that the variable sym for this solution is no longer zero, but has a zero mean value, which indicates, in accord to the theory, that the bifurcating two-torus is $R$ equivariant. This is also the reason why the period of the envelope of $E_{K}$ is half of that of $w$ and sym. The behavior of the quasiperiodic solution shows differences from that of the periodic one that mainly affects the secondary vortices near the lateral wall, which now do not appear symmetrically. In Fig. 11 we have plotted the contours of the deviation of temperature $\theta$ and the streamlines of the quasiperiodic solution at
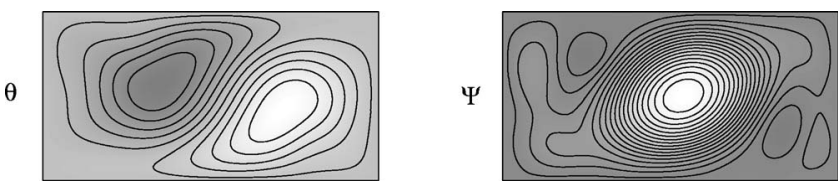

FIG. 11. Contours of the deviation of temperature $\theta$ and streamlines of the quasiperiodic solution at $\mathrm{Ra}=22000$ in an instant of time corresponding to the stretching of the roll. 
a)
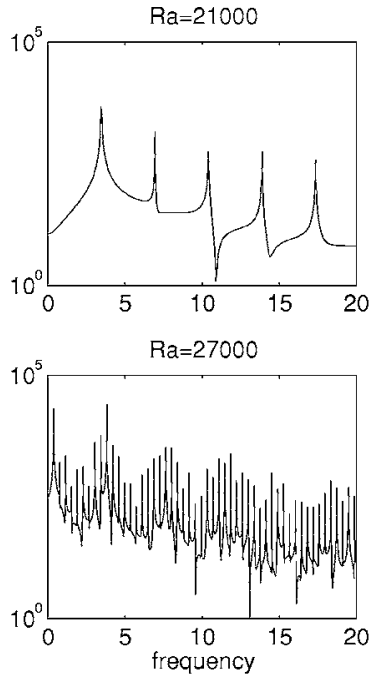

$\mathrm{Ra}=21000$

b)
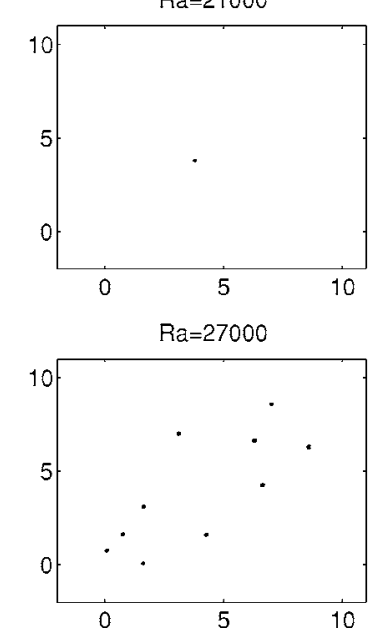
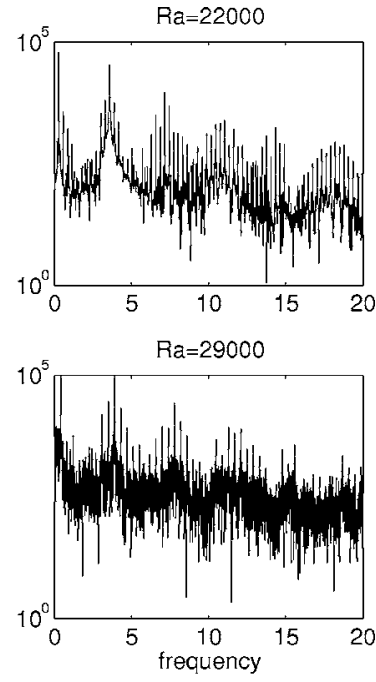

$\mathrm{Ra}=22000$

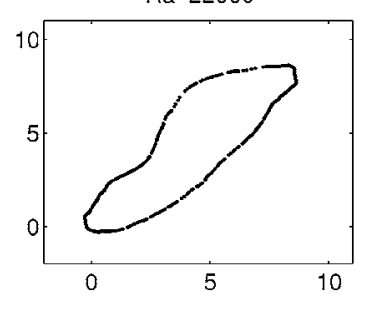

$\mathrm{Ra}=29000$

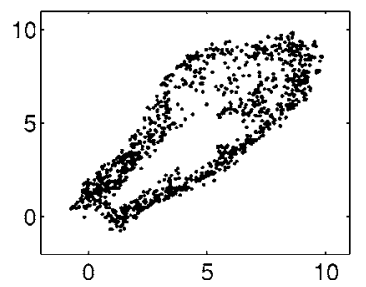

10

FIG. 12. (a) Fourier spectra and (b) Poincaré section return maps for solutions at $\mathrm{Ra}=21000,22000,27000$, and 29000 .

$R a=22000$ in an instant of time corresponding to the stretching of the roll. Here, we can clearly appreciate the symmetry breaking.

To analyze the new branch of solutions, both Fourier spectra and Poincaré return maps have been employed. To use them, it has been necessary to consider a long enough time series in order to make sure that transients have disappeared. The Poincaré section has been constructed by fixing a suitable reference value of the variable $\theta\left(P_{1}\right)$ and recording the values taken by $\beta_{n}=w\left(P_{1}\right)$ at each time that $\theta\left(P_{1}\right)$ crosses the reference value decreasing. The plot of $\beta_{n+1}$ versus $\beta_{n}$ then defines the Poincaré return map used here. For these solutions, the time interval between two successive impacts of the variable $\theta$ is associated with the basic period. In Fig. 12 we have plotted Fourier spectra of the horizontal velocity signal in the point $P_{1}$ and the Poincaré return maps for different $\mathrm{Ra}$ values. We can observe the invariant circle of the return map at $\mathrm{Ra}=22000$, corresponding to a quasiperiodic solution. We have included analogous plots for the periodic solution at $\mathrm{Ra}=21000$ in order to facilitate the identification

of the two frequencies involved. The basic frequency at Ra $=22000$ is $f_{1}=3.6$, and the secondary Hopf frequency is $f_{2}$ $=0.29$.

By decreasing the Rayleigh number the quasiperiodic solutions persist until $\mathrm{Ra} \approx 18300$, where a jump to the periodic basic solution takes place. Increasing $\mathrm{Ra}$, the system enters a quasiperiodic route to chaos. The associated quasiperiodic and periodic solutions have already been described in great detail in Ref. 31. We will describe here the main lines of the dynamics on this branch of solutions. In summary, when the Rayleigh number is increased, several intervals of frequency locking can be found associated with Neimark-Sacker bifurcations. They are of the type 1/q: namely $1: 12,1: 11$, and $1: 10$. Inside these resonance horns the stable limit cycles lose and gain stability via some typical scenarios of bifurcations of periodic solutions, ${ }^{31}$ similar, for instance, to those found in Refs. 16 and 30. Further details can be found in the quoted reference. ${ }^{31}$ Eventually, a complicated bifurcation diagram of the stable limit cycle of the 1:10 resonance horn gives rise to chaotic states for Rayleigh numbers starting from $\mathrm{Ra} \approx 28000$. In Fig. 12 we have included the Fourier spectra and the return maps of the Poincaré section corresponding to a periodic nonsymmetric cycle inside the resonance horn 1:10 $(\mathrm{Ra}=27000)$ and to a chaotic solution $(\mathrm{Ra}=29000)$.

However, when following the chaotic branch by increasing the Rayleigh number, there are small windows in which the attractor exhibits again a quasiperiodic behavior. This can be noticed in Fig. 13, where temporal evolutions of the kinetic energy $E_{K}$ are represented for different values of the Rayleigh number. An analogous behavior in a different context was observed in the bifurcation sequence leading to the breakup of an invariant torus studied in Ref. 30. For Rayleigh number $\mathrm{Ra}=35000$ a jump to another branch of solutions (see Fig. 13), coming from two successive period doubling of the basic $F$ cycle, takes place.

We have carried out a quantitative characterization of the dimensionality of the chaotic solutions by using standard nonlinear time-series analysis, ${ }^{32,33}$ on a series recorded at different preselected points of the flow. This characterization was checked to be independent of the point chosen and of the variable used, and was performed with a large time series taken from the solutions for $\mathrm{Ra}=29000,33$ 800, and 34500. For all of these cases the computed value of the maximum Liapunov exponent was positive, consistent with the chaotic character of the temporal series.

To estimate the dimension of the attractor we have used the standard method of time delays. We construct a $d$-dimensional vector out of the time series by taking $d$ values of the continuous time evolution, separated by a fixed delay time $\tau$. The idea is then to find the minimal dimension necessary to embed the resulting trajectory (without intersections). This is called the embedding dimension $d_{E}$ and defines an upper bound of the actual fractal dimension of the attractor. $^{32}$ One possible way to estimate the embedding dimension is to compute the fraction of false neighbors (points of the evolution that appear to be close to others, within a predefined distance, but only as a consequence of the low dimensionality of space). The fraction of false neighbors is a 


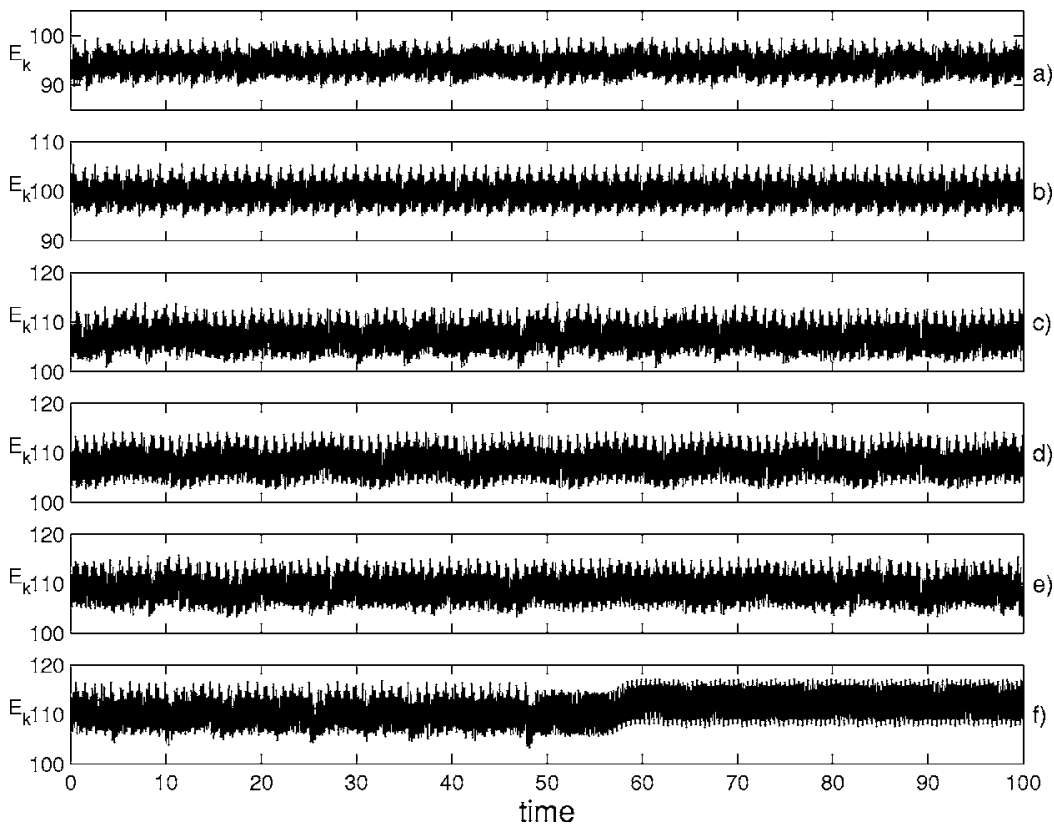

FIG. 13. Time series of the kinetic energy $E_{K}$ for solutions at (a) $\mathrm{Ra}=29000$, (b) $\mathrm{Ra}=30950$, (c) $\mathrm{Ra}$ $=33$ 800, (d) $R a=34$ 200, (e) $R a=34$ 500, and (f) $R a$ $=35000$.

decreasing function of the dimension $d$, which decays strongly around the actual value of the embedding dimension. Although the result is in principle independent of $\tau$, in practice this value must be taken carefully. A common procedure is to choose the delay time after a previous analysis of the autocorrelation function of the time series (see inset of Fig. 14). We have taken our delay time as the first zero of the autocorrelation function. This is consistent with the choice from other methods, such as the one based on the so-called average mutual information. ${ }^{32}$ Figure 14 shows the results of the false neighbor statistics obtained. Although this is only intended as an estimation of a bound for the dimension of the attractor, it is sufficient to conclude that the temporal chaos obtained in all cases is of rather low dimensionality (with an

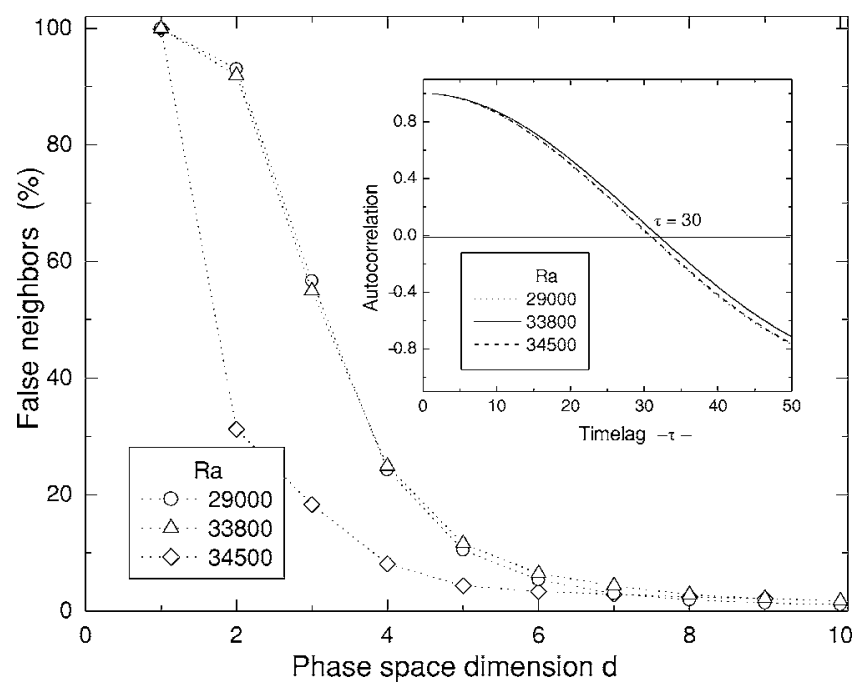

FIG. 14. Phase-space analysis of three chaotic series corresponding to the horizontal component of the velocity $u$, at a particular point of the cavity, for different Rayleigh numbers. False neighbor statistics are used for the estimation of the embedding dimension $d_{E}$. The inset shows the autocorrelation functions corresponding to the three series. embedding dimension $d_{E} \sim 6$, for the three cases considered). As a general comment it is worth remarking that in the chaotic regimes, as in the cases with simpler temporal dependence, the spatial structure of the flow remains relatively simple, with a well defined central roll.

\section{Adiabatic case}

In the adiabatic case a complex scenario of bifurcations appears by changing the Rayleigh number. To help follow the discussion, a schematic description of the branches of solutions for this case is shown in Fig. 15. Starting from the branch of $F$ cycles described in Sec. IV A, we find the Neimark-Sacker bifurcation commented on above, giving rise to quasiperiodic solutions. When the Rayleigh number is further increased, these quasiperiodic solutions are stable until $\mathrm{Ra}=2.69 \times 10^{4}$, where a new bifurcation takes place. This bifurcation is slightly subcritical, breaks the $R$ symmetry of the quasiperiodic solution, and introduces a new frequency $f_{3}$, giving rise to a three-torus. The value of this new frequency $f_{3}$ at the bifurcation point, $f_{3} \approx 0.020$, could be obtained again from the growth, out of numerical noise, of the sym indicator, which was zero in the branch $\mathrm{T}^{2}(F)$. When the

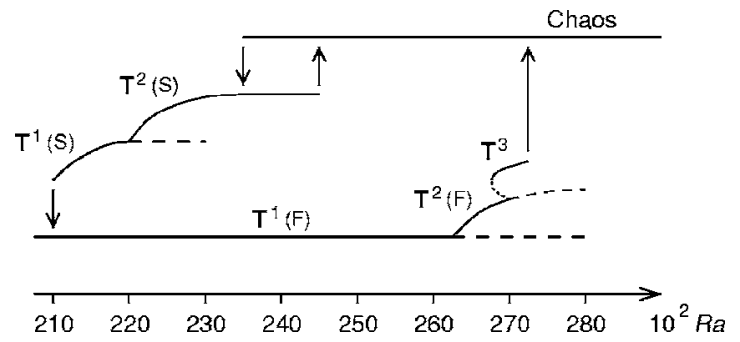

FIG. 15. Schematic diagram of the different branches of solutions for the adiabatic case. $T^{1}$ indicates a periodic solution, $T^{2}$ and $T^{3}$ indicate two- and three-tori, respectively. $F$ indicates fixed solutions and $S$ denotes $R$-equivariant orbits [e.g., $S$ cycles $\mathbb{T}^{1}(S), R$-equivariant two-tori $\mathrm{T}^{2}(S)$, etc.]. 

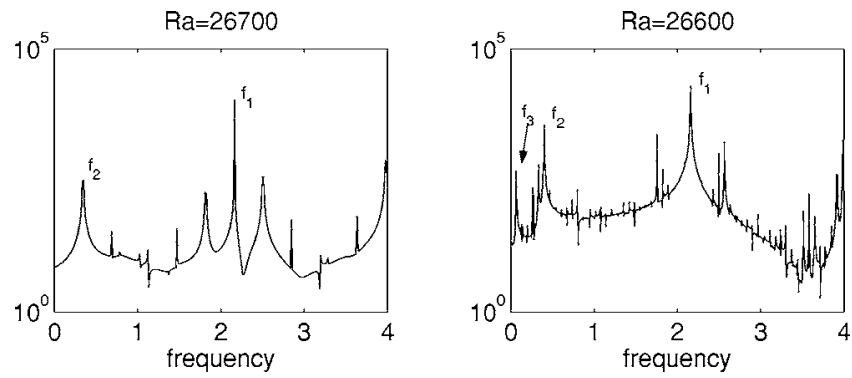

FIG. 16. Fourier spectra of $u\left(P_{1}\right)$ for the quasiperiodic solution at $\mathrm{Ra}$ $=26700$ and for the three-torus solution at $\mathrm{Ra}=26600$.

Rayleigh number decreases, the three-torus solution jumps back to the quasiperiodic two-torus at $\mathrm{Ra}=2.65 \times 10^{4}$.

In Fig. 16 we have plotted the Fourier spectra of the time series of the variable $u\left(P_{1}\right)$ for the quasiperiodic solution at $\mathrm{Ra}=26700$ and for the three-torus solution at $\mathrm{Ra}=26600$ (below the bifurcation point). Here the frequencies $f_{1}, f_{2}$, and $f_{3}$ can be clearly identified. By inspection of the Poincaré section of the three-torus solution we have discarded the possibility that $f_{3}$ could correspond to a linear combination of $f_{1}$ and $f_{2}$. The Poincaré section has been built now by fixing a proper reference value of the Nusselt number $\mathrm{Nu}$, and recording the values $\beta_{n}=w\left(P_{1}\right), \gamma_{n}=u\left(P_{1}\right)$ and the times $t_{n}$ at which $\mathrm{Nu}$ crosses the reference value while decreasing. Again, the time lag between two successive impacts is associated with the basic period. When we plot $\beta_{n}$ (or $\gamma_{n}$ ) versus $t_{n}$ for the three-torus solution, a modulated two-frequency signal is obtained as expected. The branch of stable $\mathbb{T}^{3}$ solutions does not extend very far, but several episodes of the locking of two, and even three, of the frequencies occur. Increasing further the Rayleigh number the three-torus ceases to be stable at $\mathrm{Ra}=2.72 \times 10^{4}$, where a new jump occurs, but now to a branch of chaotic states.

It is worth remarking here that the presence of a branch of stable quasiperiodic solutions involving three incommen- surate frequencies is not very common. However, it is known that three ${ }^{34}$ (or more ${ }^{35}$ ) incommensurate frequencies can coexist in a stable solution, as opposed to the most usual case of the Ruelle-Takens ${ }^{36}$ route to chaos. A transition to chaos out of a quasiperiodic solution on a three-torus, has been reported in the lateral heating of a vertical layer, ${ }^{37}$ a physical setup not very different from ours.

The nonlinear time-series analysis of the dynamics of the chaotic solutions gives results that are qualitatively similar to those of the perfectly conducting case. The temporal chaos exhibited by the system at $\mathrm{Ra}=29000$ is also of low dimensionality, with the embedding dimension $d_{E}$ estimated to be around 6. The delay time employed has been of the same order (46 t.u.) as in the conductive case. Regarding the structure of the signal it is interesting to note a peculiar, apparently random, alternation between two different types of solutions, similar to the solutions present at smaller Rayleigh numbers (see Fig. 17). The decrease of Rayleigh number starting from the chaotic solution obtained at $\mathrm{Ra}=27200$, makes the time series to lose this alternation, as one of the behaviors increasingly prevails (see Fig. 17). Near Ra $=2.40 \times 10^{4}$, the time series become very regular. We suspect that this behavior could be associated with the presence of a close, unstable branch of quasiperiodic solutions. This branch would come from a subcritical, symmetry-breaking, Neimark-Sacker bifurcation of the basic branch of $F$ cycles occurring at a value of the Rayleigh number larger than $\mathrm{Ra}_{\mathrm{NS}}=2.62 \times 10^{4}$. In fact, by changing initial conditions of the temporal integration, we have obtained at $\mathrm{Ra}=27000 \mathrm{a}$ stable periodic orbit, coexisting with the chaotic one, which could belong to the resonance horn 1:9 of the two frequencies of this "ghost" quasiperiodic solution.

Decreasing further the Rayleigh number, the signal becomes again chaotic and at $\mathrm{Ra}=2.35 \times 10^{4}$ the system evolves to another branch of solutions (see Figs. 15 and 17). The solution at this value of the Rayleigh number is quasiperiodic with two frequencies. One of these frequencies ap-

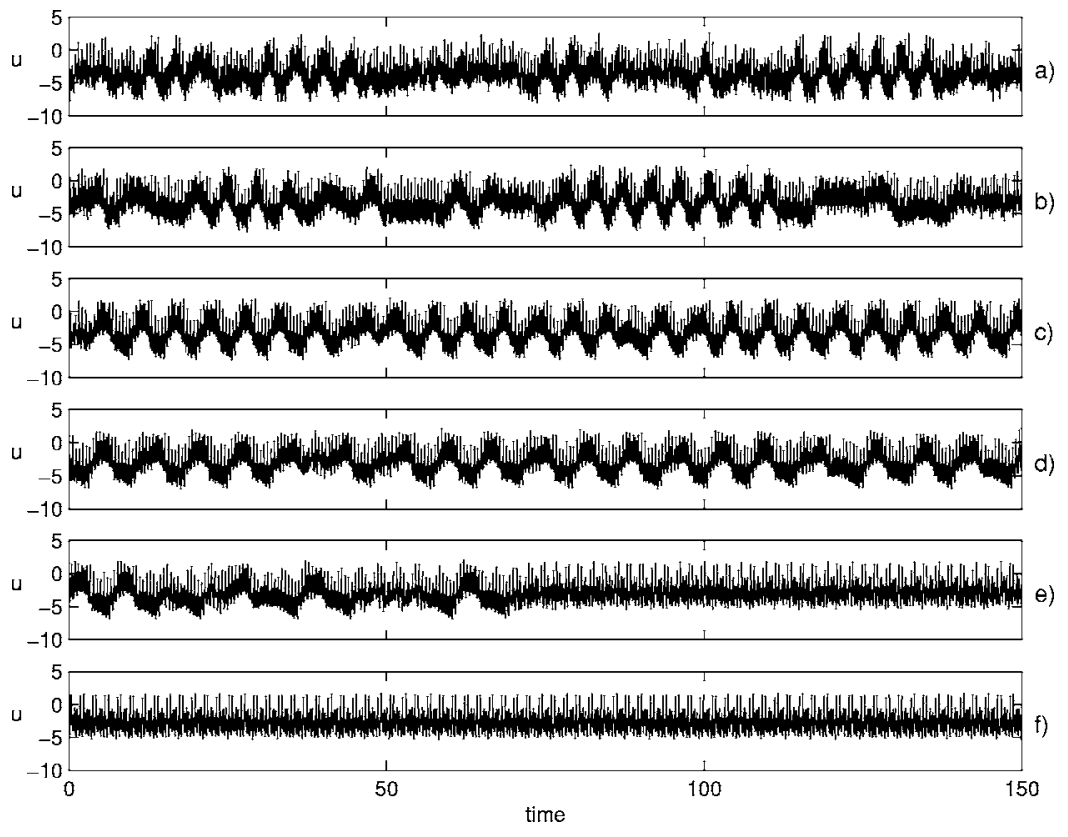

FIG. 17. Time series of the horizontal velocity $u\left(P_{1}\right)$ for solutions at (a) $\mathrm{Ra}=29000$, (b) $\mathrm{Ra}=27000$, (c) $\mathrm{Ra}$ $=25$ 000, (d) $R a=23$ 700, (e) $R a=23$ 500, and (f) $R a$ $=23000$ obtained by decreasing the Rayleigh number in the chaotic branch (see Fig. 15). 

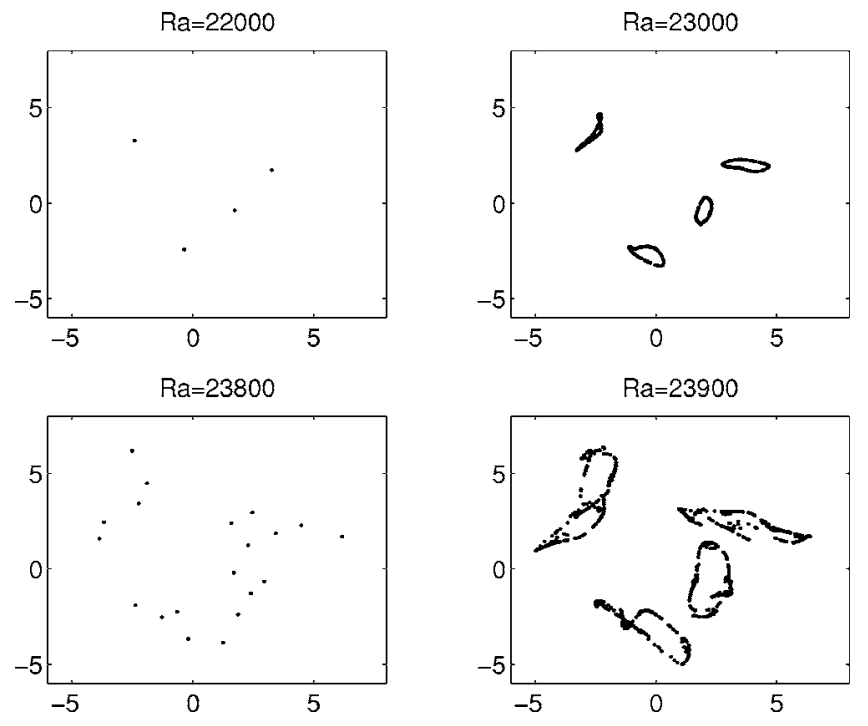

FIG. 18. Poincaré section return maps for solutions at $\mathrm{Ra}$ $=22000,23000,23800$, and 23900 .

pears to be related to the base frequency as $f^{\prime}=f_{1} / 4$, whereas the other one is new, of value $f_{3}^{\prime} \approx 0.28$. Quasiperiodic solutions $\left[\mathbb{T}^{2}(S)\right.$ in Fig. 15] are obtained until $\mathrm{Ra}=2.28 \times 10^{4}$, where a supercritical Neimark-Sacker bifurcation has taken place. The periodic solution that loses stability in this bifurcation is an $S$ cycle with frequency $f_{1}^{\prime}=f_{1} / 4\left[\mathbb{T}^{1}(S)\right.$ in Fig. 15]. The Poincaré section return maps $\left(\gamma_{n}, \gamma_{n+1}\right)$ of the periodic and quasiperiodic solutions at $\mathrm{Ra}=22000$ and 23000 are depicted in Fig. 18. The origin of this branch of solutions could be a flip bifurcation of the basic $F$ cycle maintaining the $R$ symmetry and a second flip bifurcation breaking this symmetry. However, this conjecture cannot be stated, since when the Rayleigh number is decreased further, a jump to the basic $F$ cycle is obtained at $\mathrm{Ra}=2.10 \times 10^{4}$. Notice that, as we have mentioned above, for lower values of the Rayleigh number, a period-doubling tendency had become apparent in the transients, which had contributed to the presence of an unstable fixed double-period solution.

Starting from the quasiperiodic solution obtained at $\mathrm{Ra}$ $=2.35 \times 10^{4}$, and increasing the Rayleigh number, the system enters in a resonance horn with a frequency locking $f_{3}^{\prime} / f_{1}^{\prime}$ $=1 / 5$. When the horn is exited the $\mathrm{T}^{2}$ becomes less smooth and eventually at $\mathrm{Ra}=2.44 \times 10^{4}$ a jump to the chaotic branch described above is observed. Then, there is a small range of values of the Rayleigh number where two different types of chaotic solutions coexist. In Fig. 18 we have included the Poincaré section return maps for a solution inside the quoted horn $\left(\mathrm{Ra}=2.38 \times 10^{4}\right)$ and outside the horn $(\mathrm{Ra}$ $=2.39 \times 10^{4}$ ) showing the loss of smoothness of the torus. All this behavior is also schematically shown in Fig. 15.

\section{SUMMARY AND CONCLUSIONS}

We have performed an extensive numerical study of the instabilities of natural convection in a differentially heated rectangular cavity for a fluid of low Prandtl number. The aspect ratio was chosen so that the system maintains a basic single-roll flow configuration and its instabilities lead to time-dependent behavior without breaking this basic structure. We have considered two different boundary conditions at the horizontal walls (perfectly conducting and isolating ones) and we have varied several parameters of the system, most importantly the Rayleigh number. The system has the $\mathbf{Z}_{2}$ symmetry, which has major consequences on the nature of the first solutions and their bifurcations.

The threshold value of the Rayleigh number for the Hopf bifurcation of the single-roll stationary solution presents a minimum with an aspect ratio around 2. The single roll is thus most unstable to time-dependent (single-roll) solutions for this value. The Prandtl number has been explored in a neighborhood of the value of molten germanium, $\sigma$ $=0.00715$, in order to characterize the sensitivity of the Hopf threshold value to this parameter. For the rest of the study both the Prandtl number and the aspect ratio have been fixed to the above values.

The main focus of the paper has been the elucidation of the dynamical scenario in terms of Rayleigh number as the main control parameter, and in the effects of the type of boundary conditions, either perfectly conductive or adiabatic. Varying the Rayleigh number in a broad range, we have unveiled a very rich scenario of dynamical behaviors for both boundary conditions. For small Ra values the behavior for both configurations (including the first bifurcations) are quite similar, with their steady solutions losing stability in supercritical Hopf bifurcations and giving rise to periodic $F$ cycles. The source of instability in this bifurcation is of a hydrodynamic nature. The effects of the two different thermal boundary conditions start to deviate from each other as $\mathrm{Ra}$ is further increased. Neimark-Sacker bifurcations of the $F$ cycles, subcritical in the conducting case and supercritical in the adiabatic one, give rise to quasiperiodic solutions, which, respectively, lack and possess $R$ equivariance. All the evidence from the analysis of the stratification generated in the cavity and from a detailed stroboscopic decomposition of the time evolution, seems to point to the occurrence of internal gravity waves as the instability mechanism for these secondary bifurcations. Nonetheless, the detailed response of the system to this instability is very sensitive to the type of thermal boundary conditions. A complex sequence of further bifurcations are found next, which have been characterized in detail. In the conductive case, we have obtained a transition to chaos due to torus breakdown. Before the destruction of the torus, the system passes through various resonance horns, in which the stable limit cycles lose and gain stability following several typical scenarios. ${ }^{31}$ The torus breakdown occurs via a soft transition due to loss of torus smoothness. In the adiabatic case, after the Neimark-Sacker bifurcation of the basic cycles, the dynamics seems to be dominated first by the interaction between this bifurcation, which maintains the reflection symmetry, and another Neimark-Sacker bifurcation, which breaks it. Remarkably enough, the branch of solutions is stable in the presence of three incommensurate frequencies. This coexists with a chaotic branch of solutions, which exhibits intermittence between two different behaviors. Furthermore, when the behavior of the system is analyzed decreasing the Rayleigh number from the NeimarkSacker bifurcation, we obtain some indications that a flip and 
another Neimark-Sacker bifurcation of the basic cycles (for other values of the parameters) also participate in the obtained dynamics. Then, for the considered parameters, the adiabatic case seems to be more unstable, displaying a richer bifurcation structure. In both cases it is worth remarking that chaos occurs for both boundary conditions at large Rayleigh numbers (an order of magnitude larger than the first instability), with windows of periodic behavior. The nonlinear timeseries analysis of the chaotic solutions shows that the observed chaos is relatively low dimensional.

In brief, the study of single-roll convection induced by lateral heating provides a very interesting model system to study complex dynamics, including temporal chaos, in a system with relatively simple spatial structure. The study of the instabilities and the different dynamical behaviors for the particular case studied here may be relevant to a variety of problems in materials science as an illustration of what may be expected in different applications; in particular, regarding complex temporal behaviors and the conditions in which they can appear, even if the spatial structure remains relatively simple. In many of them, however, the effect of additional instabilities inherent to the three-dimensional geometries will make the bifurcation scenario even more complex, presumably leading to an earlier occurrence of timedependent instabilities. ${ }^{4}$

\section{ACKNOWLEDGMENTS}

We would like to thank for financial support DGCYT (Spain) (Projects No. BFM2003-00657, BFM2003-07850C03-02, and BQU2003-05042-C02-02), the Comissionat per a Universitats i Recerca (Project No. 2001/SGR/00221), and the European Commission (Network Project No. HPRN-CT2002-00312). Part of the numerical results were obtained by using the CESCA and CEPBA infrastructures coordinated by the Centre de Computació i Comunicacions de Catalunya $\left(\mathrm{C}^{4}\right)$.

${ }^{1}$ P. Le Quéré and M. Behnia, "From onset of unsteadiness to chaos in a differentially heated square cavity," J. Fluid Mech. 359, 81 (1998).

${ }^{2} \mathrm{H}$. Yahata, "Stability analysis of natural convection in vertical cavities with lateral heating,” J. Phys. Soc. Jpn. 2, 446 (1999).

${ }^{3}$ J. P. Pulicani, E. Crespo del Arco, A. Randriamampianina, P. Bontoux, and R. Peyret, "Spectral simulations of oscillatory convection at low Prandtl number," Int. J. Numer. Methods Fluids 10, 481 (1990).

${ }^{4} \mathrm{~S}$. Xin and P. Le Quéré, "Linear stability analyses of natural convection in a differentially heated square cavity with conducting horizontal walls," Phys. Fluids 13, 2529 (2001).

${ }^{5}$ D. Henry and M. Buffat, "Two- and three-dimensional numerical simulations of the transition to oscillatory convection in low-Prandtl-number fluids," J. Fluid Mech. 374, 145 (1998).

${ }^{6}$ A. Juel, T. Mullin, H. Ben Hadid, and D. Henry, "Three-dimensional convection in molten gallium," J. Fluid Mech. 436, 267 (2001).

${ }^{7}$ B. Hof, A. Juel, L. Zhao, D. Henry, H. Ben Hadid, and T. Mullin, "Onset of oscillatory convection in molten gallium," J. Fluid Mech. 515, 2391 (2004).

${ }^{8}$ S. Wakitani, "Numerical study of three-dimensional oscillatory natural convection at low Prandtl number in rectangular enclosures," ASME J. Eng. Gas Turbines Power 123, 77 (2001).

${ }^{9}$ J. M. Pratte and J. E. Hart, "Endwall driven, low Prandtl number convection in a shallow rectangular cavity," J. Cryst. Growth 102, 54 (1990).

${ }^{10}$ R. Delgado-Buscalioni, E. Crespo del Arco, and P. Bontoux, "Flow transitions in a low-Prandtl-number fluid in an inclined 3D cavity," Eur. J. Mech. B/Fluids 329, 1 (2001).

${ }^{11}$ A. Y. Gelfgat, P. Z. Bar-Yoseph, and A. L. Yarin, "Stability of multiple steady states of convection in laterally heated cavities," J. Fluid Mech. 388, 315 (1999).

${ }^{12}$ W. J. Boettinger, S. R. Coriell, A. L. Greer, A. Karma, W. Kurz, M. Rappaz, and R. Trivedi, "Solidification microstructures: Recent developments, future directions," Acta Mater. 48, 43 (2000).

${ }^{13}$ A. Müller and M. Wilhelm, "Periodische Temperaturschwankungen in flüssigem InSb als Ursache schichtweisen Einbaus von Te in Kristallisierendes InSb," Z. Naturforsch. A 19A, 254 (1964).

${ }^{14}$ Numerical Simulation of Oscillatory Convection in Low-Pr Fluids: A GAMM Workshop, in Notes on Numerical Fluid Mechanics Vol. 27, edited by B. Roux (Vieweg, Braunschweig, 1990).

${ }^{15}$ Y. A. Kuznetsov, Elements of Applied Bifurcation Theory, in Applied Mathematical Sciences Vol. 112 (Springer-Verlag, New York, 2004).

${ }^{16}$ F. Marques, J. M. Lopez, and J. Shen, “A periodically forced flow displaying symmetry breaking via a three-tori gluing bifurcation and two-tori resonances," Physica D 156, 81 (2001).

${ }^{17}$ S. Hugues and A. Randiamampianina, "An improved projection scheme applied to pseudospectral method for the incompressible Navier-Stokes equations," Int. J. Numer. Methods Fluids 28, 501 (1998).

${ }^{18}$ O. Batiste, E. Knobloch, I. Mercader, and M. Net, "Simulations of oscillatory binary fluid convection in large aspect ratio containers," Phys. Rev. E 65, 016303 (2002).

${ }^{19}$ E. Meca, I. Mercader, O. Batiste, and L. Ramírez-Piscina, "Blue sky catastrophe in double-diffusive convection," Phys. Rev. Lett. 92, 234501 (2004).

${ }^{20}$ E. Meca, I. Mercader, O. Batiste, and L. Ramírez-Piscina, "Complex dynamics in double-diffusive convection," Theor. Comput. Fluid Dyn. 18, 231 (2004).

${ }^{21}$ G. E. Karniadakis, M. Israeli, and S. A. Orszag, "High order splitting methods for the incompressible Navier-Stokes equations," J. Comput. Phys. 97, 414 (1991).

${ }^{22}$ S. Zaho and M. J. Yedlin, "A new iterative Chebyshev spectral method for solving the elliptic equation $\nabla \cdot(\sigma \nabla u)=f$," J. Comput. Phys. 113, 215 (1994).

${ }^{23}$ M. E. Ermakov and X. Ruiz, "New possibilities of computer laboratory for modeling of convective processes," in Grid Generation: Theory and Applications, edited by S. A. Ivanenko and V. A. Garanzha (Russian Academy of Sciences Computing Centre, Moscow, 2002).

${ }^{24}$ C. K. Mamum and L. S. Tuckerman, "Asymmetry and Hopf bifurcation in spherical Couette flow," Phys. Fluids 7, 80 (1995).

${ }^{25}$ A. Bergeon, D. Henry, H. Benhadid, and L. S. Tuckerman, "Marangoni convection in binary mixtures with Soret effect," J. Fluid Mech. 375, 143 (1998).

${ }^{26}$ V. Frayssé, L. Giraud, S. Gratton, and J. Langou, CERFACS Technical Report No. TR/PA/03/3, 2003, Public domain software available on www.cerfacs/algor/Softs.

${ }^{27}$ I. Soibelman and D. I. Meiron, "Finite-amplitude bifurcations in plane Poiseuille flow: Two-dimensional Hopf bifurcation," J. Fluid Mech. 229, 389 (1991).

${ }^{28}$ K. Lust and D. Roose, "An adaptive Newton-Picard algorithm with subspace iteration for computing periodic solutions," SIAM J. Sci. Comput. (USA) 19, 1188 (1998).

${ }^{29}$ J. Sánchez, M. Net, B. García-Archilla, and C. Simó, "Newton-Krylov continuation of periodic orbits for Navier-Stokes flows," J. Comput. Phys. 201, 13 (2004).

${ }^{30}$ D. Aronson, M. Chory, G. Hall, and R. McGehee, "Bifurcations from an invariant circle for two-parameter families of maps on the plane: A computer-assisted study," Commun. Math. Phys. 83, 303 (1982).

${ }^{31}$ I. Mercader, O. Batiste, and X. Ruiz, "Quasi-periodicity and chaos in a differentially heated cavity," Theor. Comput. Fluid Dyn. 18, 221 (2004).

${ }^{32}$ H. D. I. Abarbanel, Analysis of Observed Chaotic Data (Springer, New York, 1996).

${ }^{33}$ H. Kantz and T. Schreiber, Nonlinear Time Series Analysis (Cambridge University Press, Cambridge, 1997).

${ }^{34}$ J. P. Gollub and S. V. Benson, "Many routes to turbulent convection," J. Fluid Mech. 100, 449 (1980).

${ }^{35}$ R. W. Walden, P. Kolodner, A. Passner, and C. M. Surko, "Nonchaotic Rayleigh-Bénard convection with four and five incommensurable frequencies," Phys. Rev. Lett. 53, 242 (1984).

${ }^{36}$ D. Ruelle and F. Takens, "On the nature of turbulence," Commun. Math. Phys. 20, 167 (1971).

${ }^{37}$ D. A. Bratsun, A. V. Zyuzgin, and G. F. Putin, "Nonlinear dynamics and pattern formation in a vertical fluid layer heated from the side," Int. J. Heat Fluid Flow 10, 481 (1990). 